In gesunden Pflanzen waren solche Partikel nicht aufzufinden. Werden Primärinfekte (Nekrosen bei Chenopodium amaranticolor und Chlorosen bei Neuseeländer Spinat) aus den Blättern mit einem Korkbohrer geeigneter Größe ausgestochen und in einem Mikro-Gewebemörser in etwas Wasser homogenisiert, so lassen sich im Überstand die gleichen Partikel nachweisen, sie fehlen jedoch vollständig in dem gesunden, zwischen den primär infizierten Stellen gelegenen Gewebe.

Die oben beschriebene Kombination von Symptomen dürfte das von uns verwendete RMV hinreichend charakterisieren; eine Verwechslung mit einem der anderen auf Rüben vorkommenden bekannten
Viren ist daher ausgeschlossen. Die Tatsache, daß sowohl in den primären Infektionsherden als auch in den Exsudaten sekundärkranker Blätter nur Partikel der abgebildeten Art und keine anderen, einem Virus ähnelnden Teilchen, aufzufinden sind, berechtigt zu dem Schluß, daß die dargestellten Partikel mit einer Länge von $700 \mathrm{~m} \mu$ die die Infektiosität tragenden Einheiten des Rübenmosaikvirus sind.

Wir danken Herrn Prof. Dr. G. S chra m m und Herrn G. B e r g e r im Max-Planck-Institut für Virusforschung, Tübingen, für die Ermöglichung bzw. Durchführung der elektronenmikroskopischen Aufnahmen sowie der Deutschen Forschungsgemein$\mathrm{s} \mathrm{ch}$ a f $\mathrm{t}$ für finanzielle Hilfe.

\title{
Experimentelle Untersuchungen über die Entstehung asexueller Klone bei der Hydromeduse Eleutheria dichotoma
}

\author{
Von C. Hauenschild \\ Aus dem Max-Planck-Irsstitut für Biologie (Abt. H a r t m a n n), Tübingen \\ (Z. Naturforschg. 11 b, 394-402 [1956]; eingegangen am 10. März 1956)
}

\begin{abstract}
Die Hydromeduse Eleutheria dichotoma pflanzt sich normalerweise sowohl geschlechtlich als auch vegetativ fort. In Klonkulturen treten jedoch als Ausnahmen sprunghaft asexuelle Medusen auf, welche die Fähigkeit zur Keimzellenbildung eingebüßt haben und sich nur noch vegetativ fortpflanzen; auch von den am Polypen entstehenden Primärmedusen ist nicht selten ein kleiner Prozentsatz bereits asexuell. In beiden Fällen erscheinen in der vegetativen Nachkommenschaft der geschlechtslosen Medusen, auch bei jahrelanger Zucht, keine sexuell differenzierten Exemplare mehr. Durch Zerstückelung und Aufzucht der Regenerate sowie durch Röntgenbestrahlung ließ sich ein derartiger Verlust der Sexualität z. T. künstlich herbeiführen. Asexuelle Individuen konnten nur durch Implantation von Normalmedusen-Gewebe wieder zur Keimzellenbildung veranlaßt werden; dabei waren die an I-Zellen besonders reichen Implantate am wirksamsten. Dagegen war eine entsprechende Einpflanzung von Polypengewebe stets erfolglos.
\end{abstract}

$\mathrm{M}$ anche niedere Metazoen (vor allem gewisse Schwämme, Hydrozoen, Bryozoen, Turbellarien und Anneliden) vermehren sich hauptsächlich vegetativ, pflanzen sich aber außerdem, sei es regelmäßig oder nur unter bestimmten Bedingungen, auch geschlechtlich fort. In allen bisher bekannten Fällen geht dabei die Potenz des Stammindividuums, Keimzellen zu erzeugen, uneingeschränkt auf alle vegetativen Nachkommen über (von Stockbildung mit polymorpher Differenzierung natürlich abgesehen); auch können bei derartigen Organismen häufig beliebige Teilstücke vollständige Individuen, die nach wie vor die sexuelle Potenz besitzen, regenerieren. Dies beruht sehr wahrscheinlich darauf, daß bei solchen Tieren der ganze Körper von diffus verteilten, undiffe- renzierten Zellen durchsetzt ist (z. B. I-Zellen der Hydrozoen, Neoblasten der Anneliden), welche omnipotent und damit auch imstande sind, sich gegebenenfalls in Keimzellen zu differenzieren. Demgemäß scheint die Fähigkeit zur vegetativen Fortpflanzung die Existenz einer determinierten Keimbahn auszuschließen.

Es sind aber auch Arten bekannt, die sich nur noch vegetativ vermehren und die Sexualität augenscheinlich vollkommen verloren haben. So pflanzt sich der sedentäre Polychaet Ctenodrilus serratus in der Nordsee ausschließlich durch Querteilung fort, während aus Neapel sexuell differenzierte, zwittrige Exemplare beschrieben worden sind. Bisher wurde meist angenommen, daß ein solcher Verlust der Sexualität 
entweder auf Mutation beruht oder überhaupt nicht irreversibel ist und eine Art Dauermodifikation darstellt.

Wir kultivieren im Laboratorium seit nunmehr 4 Jahren die Hydromeduse (nebst dem dazugehörigen Hydroidpolypen) Eleutheria dichotoma, welche sich normalerweise regelmäßig sowohl vegetativ durch Medusenknospen als auch geschlechtlich durch selbstbefruchtete Eier fortpflanzt. Jedoch fanden sich unter den vegetativen Nachkommen sexuell differenzierter (im folgenden als „normal“ bezeichneter) ${ }^{*}$ Medusen von Zeit zu Zeit einige Exemplare, die keine Keimzellen entwickelten und sich nur noch vegetativ vermehrten. Solche asexuelle Medusen wurden mehrfach einige Jahre lang gesondert weitergezüchtet; dabei erwies sich in jedem Fall die gesamte Nachkommenschaft ebenfalls als asexuell, d.h. in all diesen Klonen trat bisher keine einzige geschlechtlich differenzierte Meduse mehr auf. Offenbar war schon bei den keimzellenlosen Stammindividuen dieser permanent asexuellen Klone im Zuge der vegetativen Fortpflanzung ein irreversibler Verlust der Sexualität eingetreten. Da das vegetative Fortpflanzungs-Vermögen der Medusen durch den Verlust der Sexualität in keiner Weise beeinträchtigt wird, muß man bei den Eleutheria-Medusen entweder eine frühzeitige Sonderung des undifferenzierten Materials in Urkeimzellen und somatische I-Zellen oder einen im primär omnipotenten I-Zellenbestand gewissermaßen als partielle Differenzierung sukzessiv fortschreitenden, irreversiblen Verlust der sexuellen Potenz annehmen. In beiden Fällen kann im Verlauf der vegetativen Fortpflanzung eine Verarmung an potentiellen Keimzellen eintreten, welche schließlich zur Abschnürung einer Knospe, die nur noch asexuelle I-Zellen enthält, und damit zur Entstehung eines ausschließlich asexuellen Klons führt.

Die Entstehung einer permanent asexuellen Form, wie man sie z. B. bei Ctenodrilus aus freier Natur kennt, läßt sich somit bei Eleutheria im Laboratorium regelmäßig beobachten; experimentelle Untersuchungen an diesem Objekt schienen daher geeignet, einige neue Gesichtspunkte zu dem eingangs angedeuteten Fragenkomplex beizutragen.

\section{Material und Methoden}

Das gesamte Versuchsmaterial stammt von einer einzigen Meduse ab, die im Mai 1952 auf der Insel Rhodos, und zwar in detritusreichem Sand des Mandraki-Hafens in etwa $1 \mathrm{~m}$ Tiefe gefunden wurde. Die geschlechtlichen und vegetativen Nachkommen dieses Tieres werden seit 4 Jahren bei $18-20^{\circ} \mathrm{C}$ in Boverischalen mit Nordseewas- ser (filtriert und 2-mal bei $80^{\circ} \mathrm{C}$ sterilisiert) gezüchtet. Polypen wie Medusen bekommen als Futter zweimal wöchentlich je nach ihrer Größe 1-3 Artemialarven ( 3 Tage alt) mit einer Pipette gereicht; die frisch metamorphosierten Polypen erhalten zunächst nur kleine Artemia-Stückchen. Lediglich zu bestimmten Versuchszwekken wurden manche Medusen täglich gefüttert. Das Seewasser wird 1-2-mal in der Woche gewechselt.

Bei den Transplantations- und Regenerationsversuchen wurden die Tiere vorher durch Zusatz von Magnesiumsulfat (15 Tle. einer gesättigten Lösung auf 85 Tle. Seewasser) betäubt. Das zur Überpflanzung bestimmte Gewebestück wurde mit einem Augenskalpell aus dem Spender isoliert und im Mikromanipulator mittels einer feinen Glasnadel an der gewünschten Stelle des Empfängers solange angedrückt, bis es hier fest angewachsen war (meist nach 15-45 Min.).

Die beigegebenen Abbildungen hat Herr E. Freiberg angefertigt. Für die Betreuung der Kulturen danke ich Frl. H. Sch midt, für die Überlassung bisher unveröffentlichter Ergebnisse Frl. B. S tolt.

\section{Entwicklungsgang}

Hinsichtlich älterer morphologischer und entwicklungsgeschichtlicher Untersuchungen über die Gattung Eleutheria sei auf die Arbeit von Lengeri ch (1923) ${ }^{1}$ verwiesen.

Die eiförimge, freischwimmende Planulalarve setzt sich binnen weniger Tage auf dem Substrat fest und metamorphosiert umgehend in einen kleinen Polypen mit zunächst 3-4 unverzweigten Tentakeln, zu denen später $1-4$ weitere hinzukommen. Der basale Abschnitt des Polypen umgibt sich mit Periderm und wächst in einen meist unverzweigten oder einfach gegabelten Stolo aus, der häufig nur an der Polypenbasis auf der Unterlage haftet (Abb. 1). In der Regel bleiben die Polypen solitär; manchmal entwickelt sich an der Spitze des Stolos ein Tochterpolyp. Nur selten kommt es zu einer mehrfachen Verzweigung und Knospung des Stolos und zur Entstehung einer Kolonie von 3-4 Polypen. Die Stolonen von Individuen verschiedener Herkunft können bei Berührung miteinander verwachsen. Im Alter von etwa 1 Monat beginnen die Polypen, in der im unteren Körperdrittel gelegenen Knospungszone fortlaufend Primärmedusen zu erzeugen; einzelne Polypen konnten bis zu $1 \mathrm{Jahr}$ bei fortgesetzter Medusenknospung am Leben erhalten werden. Die Primärmedusen entstehen entweder einzeln oder zu mehreren gleichzeitig (2-4) an einem kurzen Gonostyl (Abb. 1). Die Entwicklung von der Anlage der Knospe bis zur Ablösung der Meduse dauert ca. 1 Woche. Die junge Primärmeduse besitzt meist 6 , seltener 4 oder 5 gabel-

1 H. L e n g e r i c h, Zool. Jb. Anatom. 44, 311 [1923]. 


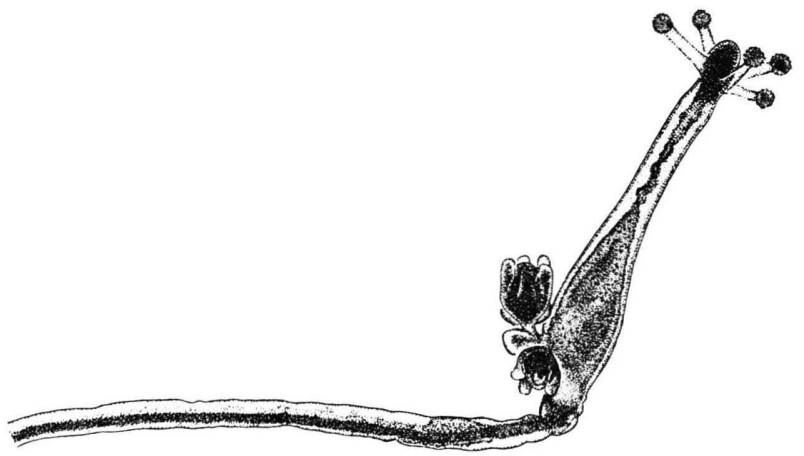

Abb. 1. Polyp (Stolo nur zu $2 / 3$ abgebildet) mit 4 Primärmedusen-Knospen an einem verzweigten Gonostyl (die jüngste noch ohne Tentakelanlagen, die älteste fast reif zur Ablösung) 20-fach vergr.

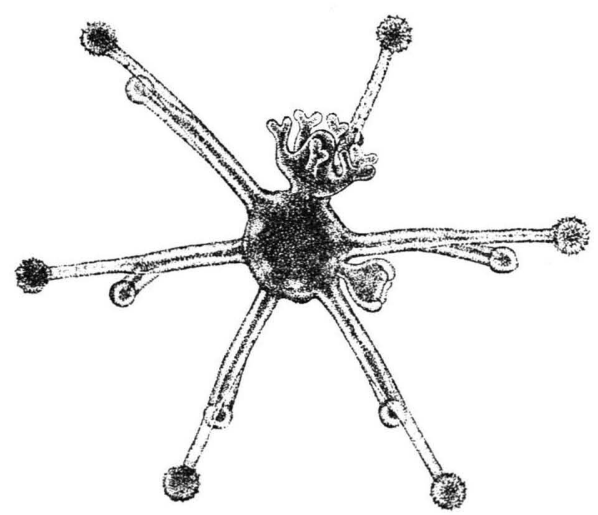

Abb. 2. Auf der Unterlage festsitzende, unreife (bzw. asexuelle) Meduse mit zwei verschieden alten Sekundärmedusen-Knospen (aborale Ansicht) 20-fach vergr.

förmig in Wehr- und Schreitast gespaltene Tentakel (Abb. 2); die ursprüngliche Tentakel-Anzahl kann zeitlebens beibehalten werden, häufig erhöht sie sich jedoch durch die Bildung accessorischer Tentakel nachträglich auf maximal 11. Etwa $1-2$ Wochen nach ihrer Lösung vom Polypen schnürt die Primärmeduse die erste Sekundär-Medusenknospe ab; bei maximaler Fütterung können mehrere Tochtermedusen (bis zu 5) gleichzeitig entstehen. Die Sekundärmedusen entwickeln sich stets im interradialen Bereich des Ringkanals (Abb. 2). Auch die Sekundärmeduse beginnt meist nach 1 Woche, durch Knospung ihrerseits eine weitere vegetative Sekundärmedusen-Generation zu erzeugen. Auf diese Weise kann jede Meduse vegetativ, vermutlich ad infinitum, als Klon weitergezüchtet werden. Die Geschlechtsreife tritt bei der Primärmeduse nach 2-4 Wochen ein. Die aus der Subumbrella in den Interradien nach oben wachsenden Keim- oder Brutschläuche haben sich dann zwischen

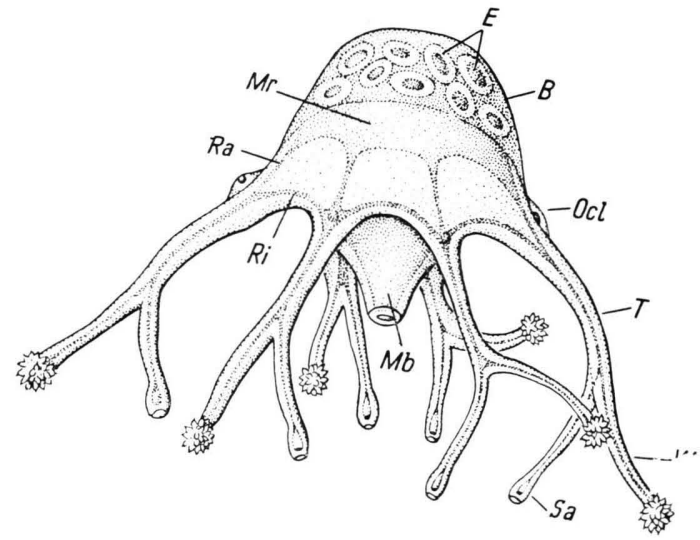

Abb. 3. Schematisierte Seitenansicht einer geschlechtsreifen Meduse (nach Hincks) 60-fach vergr. Ra = Radiärkanal, übrige Bezeichnungen wie in Abb. 4.

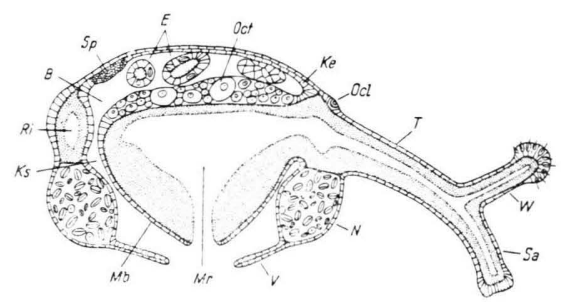

Abb. 4. Schematisierter Längsschnitt durch eine geschlechtsreife Meduse (nach Lengerich) 60-fach vergr. $\mathrm{B}=$ Brutraum, $\mathrm{E}=$ Embryonen, $\mathrm{Ke}=$ Keimepithel, $\mathrm{Ks}=$ Keimschlauch, $\mathrm{Mb}=$ Manubrium, $\mathrm{Mr}=$ Magenraum, $\mathrm{N}=$ Nesselring, $\mathrm{Ocl}=$ Ocellus, Oct $=$ Oocyte, $\mathrm{Ri}$

- Ringkanal, $\mathrm{Sa}=$ Schreitast des Tentakels, $\mathrm{Sp}=$ Spermatocyten, $\mathrm{T}=$ Tentakel, $\mathrm{V}=$ Velum, $\mathrm{W}=$ Wehrast des Tentakels.

Exumbrella und Magendach zu einem einheitlichen, von ektodermalem Epithel gebildeten Brutraum vereinigt, in dem sich gleichzeitig Oocyten und Spermien entwickeln (Abb. 3 und 4). Die nach Selbstbefruchtung aus den Eiern hervorgegangenen Planulalarven verlassen den Brutraum durch seine subumbrellaren Öffnungen. Daß die Planulae durch einen Riß in der Exumbrella frei werden, wie dies Lengerich ${ }^{1}$ angibt, kommt nur bei alten Medusen gelegentlich vor und ist als Degenerationserscheinung aufzufassen; in solchen Fällen machen die Larven nicht selten sogar ihre Metamorphose im Brutraum durch. Die Sekundärmedusen werden in der Regel schon nach etwa $1-2$ Wochen geschlechtsreif. Bei normaler Fütterung hört mit Beginn der geschlechtlichen Fortpflanzung die Erzeugung von Medusenknospen sofort auf; nur durch maximale Nahrungszufuhr läßt es sich erreichen, daß gleichzeitig Eier und Knospen gebildet werden. Ist bei prall mit Eiern und Larven ge- 
fülltem Brutraum die Knospenbildung erst einmal zum Stillstand gekommen, dann kann sie meist nur durch eine 1-2-malige Entleerung des Brutraums (Schnitt in die Exumbrella) bei gleichzeitig täglicher Fütterung wieder in Gang gebracht werden.

Da der Begriff „Generation“ in der verschiedensten Bedeutung gebraucht wird, verwenden wir zur eindeutigen Beschreibung der bei Eleutheria gegebenen Verhältnisse folgende Nomenklatur: Den vom reifen Ei bis zur geschlechtsreifen Meduse sich erstreckenden, d. h. je eine Polypen- und Medusen-,,Generation“ umfassenden Entwicklungszyklus bezeichnen wir als genetische Generation; sie dauert bei Eleutheria etwa 2 Monate. Bisher wurden 14 genetische Generationen gezüchtet. Die Polypengeneration umfaßt den Eniwicklungsabschnitt vom reiten Ei bis zur Abknospung der ersten Primärmeduse; die Medusengeneration beginnt damit, daß sich die vegetativ erzeugte Medusenknospe selbständig macht, und endet, je nach der in Betracht gezogenen Fortpflanzungsart, mit dem Eintritt der Geschlechtsreife bzw. mit der Abschnürung der ersten Tochtermeduse. Dies gilt ebenso für die vom Polypen erzeugten Primärmedusen wie für die von ihresgleichen abstammenden Sekundärmedusen.

\section{Versuche über die Determination der Medusenknospe}

Junge Primärmedusen-Knospen, die vor Anlage der Tentakelspaltung vom Polypen abgetrennt wurden, entwickelten sich niemals zu Medusen weiter. Von 52 isolierten Knospen dieser Art zerfielen 20 nach kurzer Zeit, 24 blieben indifferent und 8 entwickelten sich rückläufig zu Polypen, von denen 5 von vorneherein normal, 3 dagegen zunächst anormal waren. Diese anormalen Polypen besaßen überzählige, z. T. abnorm lange und medusenähnliche (allerdings ungespaltene) Tentakel, die teilweise weit unterhalb des Mundfeldes entsprangen (Abb. 5). Vielleicht war in der abgetrennten Knospe ein Teil des Zellmaterials schon „medusoid“ determiniert und verursachte bei der Rückentwicklung zum Polypen diese Mißbildungen. Die abnormen Differenzierungen wurden im Verlauf mehrerer Wochen rückgebildet, so daß die 3 Polypen nachträglich ein völlig normales Aussehen bekamen. Von 13 Knospen, die auf dem Stadium der ersten Andeutung einer Tentakelspaltung isoliert wurden, entwickelten sich 6 weiter zu verkleinerten, aber vollständigen Medusen, die übrigen 7 bildeten sich zurück. 19 Knospen, die bei der Abtrennung schon deutlich zweihöckerige Tentakelanlagen besaßen, ent-

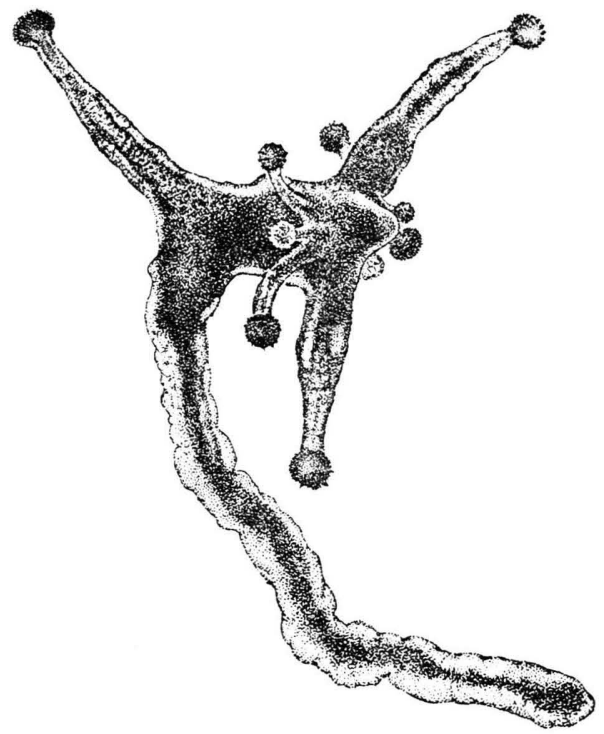

Abb. 5. Anormaler Polyp, der sich aus einer frühzeitig isolierten Primärmedusen-Knospe entwickelt hat (unten: der Stolo, rechts oben: der Mundkegel des Polypen) 35-fach vergr.

wickelten sich ausnahmslos zu kleinen Medusen weiter. Auch 2 auf diesem Stadium befindliche Knospen, die nach der Abtrennung in je zwei gleiche Hälften durchschnitten wurden, ergaben 4 kleine Medusen mit zunächst je 3 Tentakeln. Dagegen bildeten sich 2 Knospen, denen die gespaltenen Tentakelanlagen abgeschnitten wurden, zurück und entwickelten sich nicht weiter. Der Zeitpunkt, an dem die Organisation der entstehenden Primärmeduse fest determiniert ist, kann also offenbar nicht von der Größe, sondern nur von einem bestimmten Differenzierungszustand der Knospe abhängig sein, welcher etwa bei Anlage der Tentakelspaltung erreicht wird. Ob die Tentakel selbst einen determinierenden Einfluß ausüben, konnte bisher noch nicht geklärt werden.

Sekundärmedusen-Knospen, die vor Erreichen des kritischen Stadiums abgeschnitten wurden, entwikkelten sich erwartungsgemäß niemals zu Polypen, sondern zerfielen ausnahmslos nach einiger Zeit. Isolierte Knospen mit gespaltenen Tentakelanlagen differenzierten sich auch bei den Sekundärmedusen normal weiter.

\section{Der spontane Verlust der Sexualität}

Die kontrollierte Aufzucht einer größeren Anzahl von Primärmedusen der 7. genetischen Generation ergab, daß bereits von diesen direkt am Polypen entstandenen Medusen ein kleiner, von Fall zu Fall 
wechselnder Prozentsatz asexuell ist. Die Sicherheit, daß bei einer Meduse wirklich ein irreversibler Sexualitätsverlust und nicht etwa nur eine Verzögerung der Geschlechtsreife vorliegt, haben wir auf Grund entsprechender Erfahrungen dann als gegeben erachtet, wenn nicht nur die betreffende Meduse selbst, sondern auch eine ihrer ersten Tochtermedusen mindestens einen Monat lang keine Andeutung einer Keimzellenentwicklung erkennen ließ und sich während dieser Zeit fortgesetzt vegetativ vermehrte. Ein Brutraum kommt bei asexuellen Individuen, auch im adulten Stadium, nicht zur Entwicklung (Abb.6).

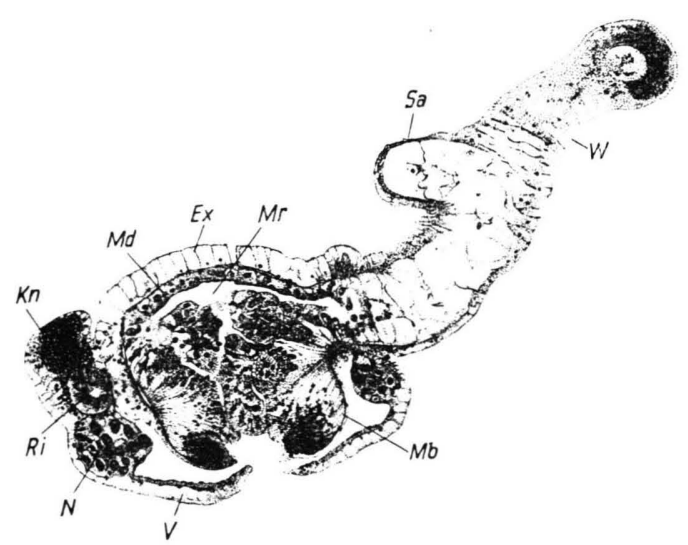

Abb. 6. Längsschnitt durch eine erwachsene asexuelle Meduse (Fixierung: Bouin, Färbung: Eisenhämatoxylin n. Heidenhain) 70 -fach vergr. $\mathrm{Ex}=$ Exumbrella, $\mathrm{Kn}=$ junge Knospe, $\mathrm{Md}=$ Magendach, übrige Bezeichnungen wie in Abb. 4.

Von 292 Primärmedusen, die von 22 verschiedenen Polypen abstammten, erwiesen sich $20(7 \%)$ als asexuell. Von den 22 kontrollierten Polypen erzeugten 9 ausschließlich normale Medusen und 13 normale und asexuelle Medusen gemischt. Hinsichtlich der Reihenfolge, in der normale und asexuelle Medusen gebildet wurden, war keine Regelmäßigkeit festzustellen. In der vegetativen Nachkommenschaft eines Polypen wurden im Höchstfall 20\% asexuelle Medusen gefunden. Ein leider nicht im einzelnen protokollierter Fall ließ allerdings erkennen, daß u. U. auch ein wesentlich höherer Anteil an asexuellen Primärmedusen (etwa 50-60\%) auftreten kann; doch ist dies selten, und die besonderen Bedingungen hierfür sind bisher nicht bekannt.

Die ersten Beobachtungen über das Auftreten asexueller Individuen wurden an Sekundärmedusen bereits in der ersten genetischen Generation gemacht. In der aus dem Stammexemplar vegetativ hervorgegangenen Sekundärmedusen-Klonkultur fiel nach einigen Monaten eine zunehmende Verarmung an geschlechtsreifen Individuen auf. Diese Erscheinung ist, wie die späteren Untersuchungen ergaben, darauf zurückzuführen, daß die normalen Medusen bei gewöhnlicher Ernährung häufig nur eine Tochtermeduse hervorbringen, bevor sie geschlechtsreif werden, und dann nur noch Eier und Spermien erzeugen, wohingegen die asexuellen Medusen während ihres ganzen Lebens fortlaufend Tochtermedusen abknospen und dadurch einen Selektionsvorteil besitzen. Da wir beim Umsetzen der Kulturen aus der Masse der Individuen immer nur einige Exemplare zur Weiterzucht willkürlich ausgewählt hatten, war es auf diese

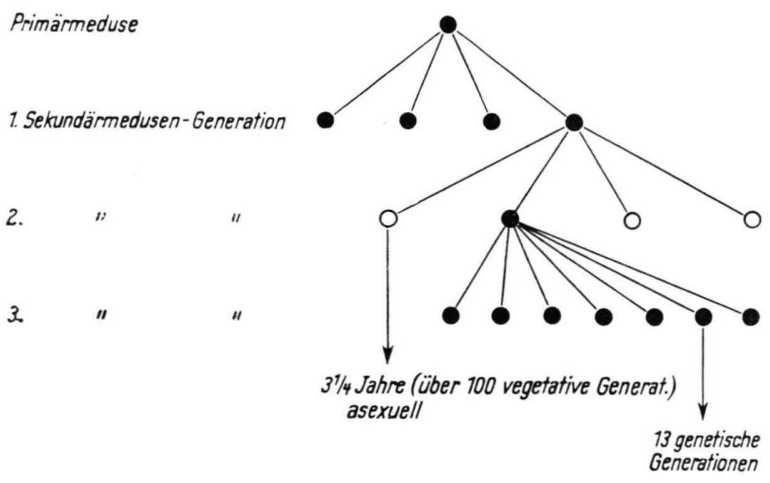

Abb. 7. Stammbaum des ersten asexuellen Klons. $\bullet=$ normale, $=$ asexuelle Meduse, weitere Erklärung im Text.

Weise zwangsläufig zu einem Überhandnehmen der asexuellen Tiere gekommen. Um etwaige RegelmäBigkeiten in der Entstehung keimzellenloser Medusen zu erfassen, wurden mehrere Versuche mit Einzelkulturen unternommen. Ein etwas extremer Fall dieser Art ist in Abb. 7 wiedergegeben. Das Auftreten asexueller Individuen erweckte jedoch in den meisten Fällen den Eindruck völliger Regellosigkeit. Niemals erzeugte eine normale Meduse ausschließlich asexuelle Tochterindividuen; vielmehr entstanden diese stets zwischen einer wechselnden Anzahl normaler Geschwister. Auch die Reihenfolge, in der normale und asexuelle Nachkommen hervorgebracht wurden, war von Fall zu Fall verschieden. Von der ersten asexuellen Meduse der 2. SekundärmedusenGeneration in Abb. 7 stammt ein Klon ab, der bereits $3^{1 / 4}$ Jahre lang vegetativ weitergezüchtet wurde (1000-2000 Individuen), ohne daß in ihm bisher eine sexuell differenzierte Meduse aufgetreten ist. Außer diesem ersten asexuellen Klon haben wir noch weitere 7 von asexuellen Medusen (teils Primär-, teils Sekundärmedusen) abstammende Klone 1 bis 
2 Jahre lang kultiviert; auch in diesen Klonen trat keine einzige geschlechtsreife Meduse in Erscheinung. Um festzustellen, ob nach einer bestimmten Anzahl vegetativer Generationen schließlich alle Sekundärmedusen ihre Sexualität verlieren, haben wir von 20 Primärmedusen jeweils die erste Sekundärmeduse, von dieser wieder die erste Tochtermeduse usf. bis zur 40. Sekundärmedusen-Generation weitergezüchtet (Versuchsdauer: 12-14 Monate). Die Ergebnisse sind in Abb. 8 graphisch dargestellt. 3 von den 20 Primärmedusen waren bereits selbst asexuell; von den übrigen 17 Stämmen verloren 3 innerhalb der ersten 7 Generationen, 2 in der 20 . und 21. und

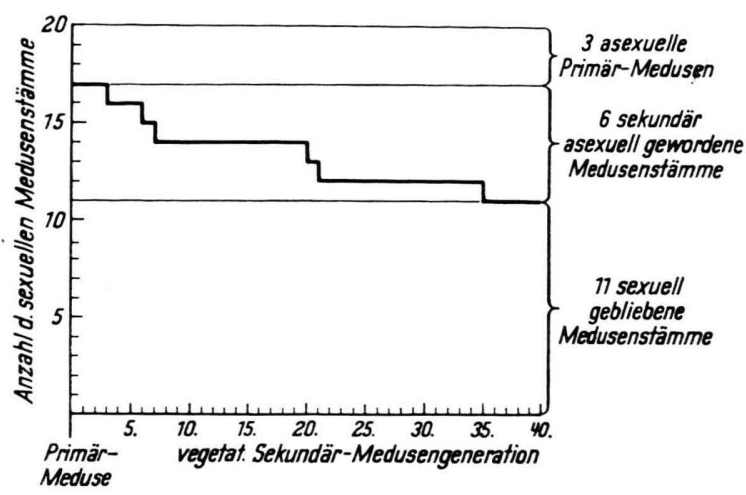

Abb. 8. Sexualitätsverluste bei 20 über 40 vegetative Sekundärmedusen-Generationen gezüchteten Stämmen; Erklärung im Text.

einer in der 35. Sekundärmedusen-Generation, insgesamt also 6, im Verlauf der vegetativen Fortpflanzung ihre Sexualität. In den übrigen 11 Stämmen erzeugten alle Medusen ohne Ausnahme bis zur 40. Generation unverändert normale Keimzellen*. Auch dieser Versuch läßt kaum eine Regelmäßigkeit in der Entstehung asexueller Medusen erkennen; lediglich eine gewisse Häufung der Sexualitätsverluste in den ersten Generationen (3 Verluste in 7 Generationen) gegenüber einer relativen Stabilität in den späteren Generationen (nur 3 Verluste in 33 Generationen) fällt auf.

\section{Versuche, den Verlust der Sexualität künstlich herbeizuführen}

Im Gegensatz zum Polypen ist bei der Meduse von Eleutheria nicht jedes beliebige Teilstück zur Regeneration eines Ganztieres befähigt. Stücke, welche Material aus der Ocellus-Region enthalten (Abb.

* Anm. b. d. Korr.: Inzwischen ist noch ein weiterer Stamm in der 53. Sekundärmedium-Generation asexuell geworden.
4), ergänzen meistens die fehlenden Teile und ergeben wieder vollständige, wenn auch manchmal etwas verkrüppelte Medusen; dagegen regenerieren z. B. isolierte Manubrien, Nesselringstücke und Tentakel (Abb. 4 und 6) nichts, obgleich sie lange am Leben bleiben. Um festzustellen, ob durch Zerstückelung und anschließende Aufzucht der Regenerate künstlich asexuelle Medusen erhalten werden können, zerteilten wir 20 sexuell noch nicht differenzierte, junge Primärmedusen der Länge nach in $4-6$ möglichst gleichartige Teilstücke, von denen jedes 1 bis 2 Tentakel mitbekam. Von den insgesamt 97 derartigen Stücken gingen 7 vorzeitig ein, die übrigen 90 konnten zu vollständigen Medusen aufgezogen werden. Eigentümlicherweise brachten die regenerierten Individuen selbst manchmal keine Keimzellen zur Entwicklung, obwohl sie normale Tochtermedusen erzeugten und somit die sexuelle Potenz keineswegs verloren hatten. Deshalb ließ sich bei den Regenerationsversuchen ein sicheres Ergebnis nur an den vegetativen Nachkommen des regenerierten Individuums gewinnen. In 14 von den 20 Fällen lieferten alle von einer Meduse stammenden Teilstücke wieder normale Medusen. In 2 Versuchen ergaben alle Regenerate asexuelle Tiere; offenbar waren hier 2 schon vor Versuchsbeginn als asexuell determinierte Primärmedusen aufgeteilt worden. In 4 Fällen regenerierten die Teilstücke einer Meduse teils normale, teils asexuelle Individuen, und zwar waren in 2 Fällen 4 Abkömmlinge normal und 1 asexuell, in einem Fall 2 normal und 1 asexuell und in einem weiteren Fall 2 normal und 2 asexuell. In 5 von den insgesamt 90 Teilstücken war somit sekundär, wohl als Folge der Regenerationsvorgänge, ein Verlust der Sexualität eingetreten.

Außer durch Regeneration versuchten wir auch durch UV- und Röntgenbestrahlung, die Bildung asexueller Medusen experimentell zu erreichen. UV wurde von den Tieren sehr schlecht vertragen, und sehr kleine, gerade noch nicht letale Dosen beeinflußten die Fähigkeit zur Keimzellenbildung in keinem Fall. Nach schwacher UV-Bestrahlung fiel lediglich auf, daß sich die Tochtermedusen sehr viel häufiger, als dies sonst der Fall ist, nicht zum normalen Zeitpunkt ablösen, sondern am Mutterindividuum hängenbleiben und hier ihre volle Größe und mitunter sogar die Geschlechtsreife erreichen. Dagegen traten nach Röntgenbestrahlung gehäuft asexuelle Medusen auf. Von 42 geschlechtsreifen Sekundärmedusen wurde die eine Hälfte mit 4000 R bestrahlt, die andere Hälfte als unbestrahlte Kontrolle verwendet. 
Um von diesen 42 Tieren Tochtermedusen zu erhalten, mußte zunächst bei allen der Brutraum künstlich entleert werden. Von jedem der Versuchs- und Kontrolltiere wurden dann bis zu 3 Tochtermedusen aufgezogen. Von den 63 vegetativen Nachkommen der 21 unbestrahlten Kontrollmedusen gingen 2 vorzeitig ein, die übrigen 61 wurden durchweg geschlechtsreif. Es ist auffallend, daß bei den Kontrolltieren somit kein einziger spontaner Sexualitätsverlust zu beobachten war. Von den 21 bestrahlten Medusen erhielten wir 51 Tochterindividuen, von denen 24 vorzeitig starben. Im Gegensatz zu der sofort sichtbaren UV-Schädigung traten die letalen Folgen der Röntgenbestrahlung erst nach Wochen und z. T. noch in der nächsten vegetativen Generation in Erscheinung. Unter den 27 überlebenden Tochtermedusen der bestrahlten Tiere befanden sich nur 11 normale, d. h. sexuell differenzierte; die übrigen 16 , also mehr als die Hälfte, erwiesen sich als asexuell. Ob diese strahleninduzierten Sexualitätsverluste auf einer selektiven Zerstörung des potentiellen Keimzellenmaterials oder auf einer allgemeinen Schädigung durch die Bestrahlung und damit vielleicht auf einem ähnlichen Prinzip beruhen wie der durch Regeneration erzielte Effekt, läßt sich vorläufig nicht entscheiden. Von den 21 bestrahlten Medusen lieferten 9 entweder keine oder nur vorzeitig letale Tochtermedusen; von den übrigen 12 ergaben (jeweils außer letalen Nachkommen) 3 ausschließlich normale, 4 nur asexuelle und 5 teils normale, teils asexuelle Tochterindividuen.

\section{Transplantationen von normalem Gewebe in asexuelle Medusen}

Auf Grund der bisher mitgeteilten Befunde war es unwahrscheinlich, daß bei den asexuellen Medusen die Potenz zur Erzeugung von Keimzellen durch eine modifikatorische und somit prinzipiell reversible Veränderung der inneren physiologischen Bedingungen lediglich unterdrückt ist; vielmehr sprach das völlig regellose und sprunghafte Auftreten asexueller Individuen sowie die absolute Konstanz des geschlechtslosen Zustandes mehr dafür, daß dem Verschwinden der Sexualität ein Verlust (bei der Knospung) oder ein „Aussterben“ (z. B. bei Regeneration oder Bestrahlung) des bei der vegetativen Fortpflanzung entbehrlichen potentiellen Keimmaterials zugrunde liegt. Diese Überlegung führte zu einer Reihe von Versuchen, asexuelle Individuen durch Implantation kleiner Gewebestücke von normalen Medusen oder Polypen wieder zur Erzeugung von Keimzellen zu veranlassen.
Zunächst implantierten wir größere, komplexe Teilstücke normaler Medusen, wie sie auch bei den Regenerationsversuchen verwendet worden waren, in Medusen des bisher seit $3^{1 / 4}$ Jahren rein asexuellen Klons. Hierbei wurden die Implantate in einem seitlich an der Empfängermeduse angebrachten Einschnitt eingepflanzt. Versuche, bei denen innerhalb von 1-2 Monaten nach der Transplantation in der ehemals asexuellen Meduse Eier gebildet wurden, bezeichnen wir als positiv $(+)$, Versuche, in denen die Entwicklung von Geschlechtsprodukten unterblieb, als negativ (-). Von 21 Implantaten, die aus ganz jungen und sexuell noch nicht differenzierten Primärmedusen stammten, waren $14+$ und 7 -; die Spendertiere wurden stets als Kontrolle bis zur Geschlechtsreife aufgezogen, um die Möglichkeit, daß sich ein primär asexuelles Individuum unter ihnen befand, auszuschließen. 23 Implantate von sexuell bereits differenzierten Medusen lieferten in 13 Fällen ein positives und in 10 Fällen ein negatives Resultat. Bei einigen dieser Versuche führte die Implantation zur Entstehung von Doppelbildungen mit 2 getrennten Manubrien. In solchen Fällen war mehrfach deutlich zu erkennen, daß die Geschlechtsprodukte, die in der aus normalen und asexuellem Gewebe bestehenden Chimaere zur Entwicklung kamen, ausschließlich aus dem Implantat stammten. Die Sexualität „induzierende“ Wirkung der Implantation besteht also offenbar nicht darin, daß eine in der asexuellen Meduse latent noch vorhandene sexuelle Potenz durch den Kontakt mit Normalgewebe physiologisch reaktiviert wird; vielmehr handelt es sich dabei augenscheinlich um einen Ersatz des in der asexuellen Meduse nicht mehr vorhandenen potentiellen Keimzellmaterials aus dem Implantat. Kontrollversuche, in denen Gewebe von asexuellen Medusen eingepflanzt wurde, waren stets negativ.

Auch ganz junge Primärmedusen-Knospen $(1+$, $11-)$ und Gonostyle $(1+, 9-)$ waren, in asexuelle Medusen implantiert, prinzipiell imstande, Keimzellen zu liefern . Dagegen erwiesen sich 42 Transplantationen von Polypengewebe in asexuelle Medusen durchweg als wirkungslos. 13 von den 42 Implantaten waren Stolonenstücke, 14 stammten aus dem Knospungsbereich und 15 aus den übrigen Körperregionen (Abb. 1). Die Polypenstücke wuchsen in den Medusen gut ein, ohne sich allerdings ortsgemäß umzudifferenzieren; so blieben z. B. eingepflanzte Sto-

- Diese Versuche wurden von Frl. B. S t o l t im Rahmen einer z. Z. noch nicht veröffentlichten Dissertation unternommen. 
lonenstücke oder orale Teile mit den für die Polypen charakteristischen ungespaltenen Tentakeln unverändert herkunftsgemäß erhalten und wurden erst nach längerer Zeit nach und nach eingeschmolzen. Auch eine asexuelle Sekundärmedusen-Knospe, die 3 Tage lang mit einem Polypen künstlich zur Verwachsung gebracht worden war, entwickelte daraufhin keine Geschlechtsprodukte. Nun muß ja an sich die Potenz zur Keimzellenbildung im Polypen, zumindest latent, bereits vorhanden sein; die Versuche zeigen jedoch, daß die sexuelle Potenz des Polypenimplantats durch den Kontakt mit ausdifferenziertem Medusengewebe nicht aktivierbar ist. Man wird daher annehmen müssen, daß die Keimzellenpotenz im Polypen blockiert ist und nur unter den spezifischen entwicklungsphysiologischen Bedingungen, wie sie allein in der entstehenden Primärmedusen-Knospe (einschließlich Gonostyl) gegeben sind, aktiviert wird, so daß sie erst von diesem Entwicklungsstadium an durch Transplantation übertragen werden kann.

Außer diesen groben, einer ersten Orientierung dienenden Überpflanzungs-Versuchen wurden 215 Transplantationen kleinster Gewebestücke (50 bis $150 \mu)$ aus verschiedenen Körperregionen normaler, sexuell noch nicht differenzierter Medusen auf das Manubrium oder Velum asexueller Individuen durchgeführt (Abb.6) 2. Durch diese Versuche sollte vor allem festgestellt werden, ob die potentiellen Keimzellen in bestimmten Teilen des Medusenkörpers lokalisiert sind. Die Ergebnisse sind in der nebenstehenden Übersicht zusammengefaßt.

Genauere Untersuchungen über die Verteilung der I-Zellen in der Eleutheria-Meduse sind erst im Gange; es ist aber schon jetzt ziemlich sicher, daß in den Implantaten, die in asexuellen Medusen am häufigsten Keimzellen lieferten (Ocellus-Region, Nesselring), die Hauptmasse der I-Zellen konzentriert ist, während die Stücke, deren Übertragung keine positiven Ergebnisse brachte (Tentakel, ManubriumSpitze), keine oder nur sehr wenige I-Zellen enthalten. Auch das eine mit regenerierendem Tentakel erzielte positive Ergebnis führt zu der Vermutung, daß die potentiellen Keimzellen entweder mit den I-Zellen identisch oder mit ihnen vergesellschaftet sind. Andererseits haben wir aber zwischen asexuellen und normalen Medusen in bezug auf den Gehalt an IZellen bisher morphologisch keinen Unterschied feststellen können.

Von den 39 asexuellen Medusen, die nach Einpflanzung kleiner Normalgewebe-Stücke zur Keimzellenbildung übergingen, trat bei 25 Tieren inner-

\begin{tabular}{|c|c|c|}
\hline $\begin{array}{l}\text { An- } \\
\text { zahl }\end{array}$ & Herkunft der Implantate & Ergebnis \\
\hline 30 & Ocellusregion & $13(=43 \%)+17-$ \\
\hline 30 & Nesselring & $11(=37 \%)+19-$ \\
\hline 30 & $\begin{array}{l}\text { Exumbrella } \\
\text { (zentraler Bereich) }\end{array}$ & $9(=30 \%)+21-$ \\
\hline 14 & $\begin{array}{l}\text { Interradiale } \\
\text { Ringkanalregion }\end{array}$ & $2(=14 \%)+12-$ \\
\hline 30 & Manubrium-Basis & $3\left(=10^{\circ} \%\right)+27-$ \\
\hline 15 & Regenerierender Tentakel & $1(=7 \%)+14-$ \\
\hline 37 & $\begin{array}{l}\text { Tentakel (25 Basis, } \\
6 \text { Schreitast, } 6 \text { Wehrast) }\end{array}$ & alle $37-$ \\
\hline 29 & Manubrium-Spitze & alle $29-$ \\
\hline
\end{tabular}

halb von 4 Wochen, bei 11 Tieren 5-6 Wochen und bei 3 Tieren $7-10$ Wochen nach der Implantation die Geschlechtsreife ein. Interessant ist der Vergleich zwischen 3 Nesselring-Implantaten, von denen 2 am Velum und 1 am Manubrium eingepflanzt waren. In allen 3 Fällen wurden vom Tag der Implantation an laufend alle von der Empfänger-Meduse vegetativ erzeugten Tochterindividuen isoliert, um festzustellen, innerhalb welcher Zeit nach der Implantation die erste normale Tochtermeduse gebildet wird. Bei den Medusen mit dem am Velum festgewachsenen Implantat war dies schon nach 10 bzw. 13 Tagen der Fall, d. h. nach dieser Zeit waren potentielle Keimzellen aus dem Implantat, sei es durch Wanderung oder Transport, in die Ringkanal-Region und von da aus in eine Medusenknospe gelangt. Dagegen trat bei der Meduse, der ein Stück Nesselring am Manubrium implantiert worden war, die erste normale Tochtermeduse erst nach 5 Wochen auf.

Die vermutlich mannigfachen Ursachen dafür, daß Implantate, deren sexuelle Potenz prinzipiell erwiesen ist, trotzdem in vielen Fällen negative Resultate ergaben, sind im einzelnen bisher nicht bekannt.

\section{Besprechung der Ergebnisse}

Es ist zwar, streng genommen, nicht zu beweisen, daß der bei den Medusen von Eleutheria sporadisch auftretende Verlust der Sexualität irreversibel ist; doch scheint uns die Tatsache, daß in 8 von asexuellen Individuen abstammenden Klonen während mehrerer Jahre kein geschlechtlich differenziertes Exemplar aufgetreten ist, während sich andererseits in 11 unter denselben Bedingungen existierenden Normalklonen die Sexualität über 40 vegetative Sekundärmedusen-Generationen unverändert erhalten hat, zu einer derartigen Annahme zu berechtigen. Da es zum 
Wesen einer Modifikation (auch einer Dauermodifikation) gehört, prinzipiell reversibel zu sein, ist dieser Begriff auf das beobachtete Phänomen nicht anwendbar. Der Sexualitätsverlust tritt innerhalb von Klonen, d. h. nicht im Zusammenhang mit der geschlechtlichen Fortpflanzung auf; da er überdies relativ häufig ist, kann er wohl kaum auf mutative Veränderungen im Genom zurückgeführt werden. Vielmehr scheint dem Verlust der Sexualität bei Eleutheria ein Vorgang zugrunde zu liegen, der eine gewisse Parallele z. B. in dem ebenfalls irreversiblen Verlust des Chloroplasten bei Euglena hat; hier wie dort geht dem Organismus ohne letale Folgen ein Bestandteil unersetzlich verloren, welcher bei der ungeschlechtlichen Fortpflanzung nur aus seinesgleichen entstehen, nicht aber von anderen Teilen regeneriert werden kann. Für eine derartige Erscheinung hat sich bisher kein geeigneter Begriff eingebürgert.

Es ist einigermaßen sicher, daß die Keimzellen der Hydrozoen aus I-Zellen entstehen. Da es bei Eleutheria keine Polypen gibt, die ausschließlich asexuelle Medusen erzeugen, darf man annehmen, daß die I-Zellen des Polypen stets noch sämtliche Potenzen besitzen. Bei der Bildung einer PrimärmedusenKnospe müssen jedoch alle daran beteiligten I-Zellen des Polypen irgendeine Differenzierung durchmachen, bei der sie ihre „vegetative Polypenpotenz" irreversibel verlieren und statt dessen ihre bisher ruhende Medusen- und Keimzellenpotenz entfalten. Die Keimzellenpotenz schließt natürlich sämtliche Potenzen, also auch die der Polypenform, ein; doch sind diese Potenzen gewissermaßen blockiert und können nicht bei der Regeneration oder vegetativen Vermehrung (,vegetative Potenz"), sondern nur auf dem Wege der geschlechtlichen Fortpflanzung und Embryonalentwicklung realisiert werden. Den Zeitpunkt, an dem die genannte Differenzierung der I-Zellen vollzogen ist, haben wir durch die Isolierungsversuche mit jungen Primärmedusen-Knospen annähernd bestimmt. Doch harren hinsichtlich dieser entwicklungsphysiologischen Grundlagen des Generationswechsels Polyp/Meduse noch viele interessante Probleme der Bearbeitung.
Für einen Versuch, den Verlust der Sexualität bei den Eleutheria-Medusen zu erklären, scheinen uns vornehmlich die beiden folgenden Möglichkeiten zu bestehen:

1. Alle I-Zellen besitzen nach Entfaltung ihrer Medusenpotenz zunächst auch die sexuelle Potenz, können dieselbe aber unter bestimmten inneren Bedingungen nach und nach verlieren; aus diesem Grunde enthalten manche Medusen nach einiger Zeit zwei Sorten von I-Zellen, solche, die noch die sexuelle Potenz besitzen, und solche, die sie bereits verloren haben. Es besteht bisher kein Anhaltspunkt dafür, daß sich die beiden Sorten morphologisch voneinander unterscheiden lassen. Sobald die asexuellen I-Zellen stark in der Úberzahl vorhanden sind, kann es rein zufallsgemäß zur Abknospung von Tochtermedusen kommen, an deren Aufbau keine I-Zellen mit sexueller Potenz mehr beteiligt und die infolgedessen asexuell sind. Asexuelle Primärmedusen kommen dadurch zustande, daß bei der Differenzierung der Medusenknospe am Polypen infolge besonderer Umstände die sexuelle Potenz aller beteiligten I-Zellen mit einem Schlag verlorengeht.

2. Die I-Zellen des Polypen differenzieren sich bei der Medusenbildung von vorneherein in zwei Zellsorten, in medusoide I-Zellen ohne sexuelle Potenz und in Urkeimzellen. Werden nun z. B. bei der Erzeugung der Geschlechtsprodukte mehr Urkeimzellen verbraucht, als durch Vermehrung in der gleichen Zeit nachgeliefert werden, so kann dies zu einer Verarmung an Urkeimzellen und schließlich zur Abknospung asexueller Tochterindividuen führen. Bei den asexuellen Primärmedusen müßte eine Störung des einmaligen Differenzierungsprozesses angenommen werden.

Eine Entscheidung zwischen den beiden Deutungen ist z. Z. nicht möglich. Im Falle der Deutung 2 wäre es berechtigt, bei Eleutheria von einer auf die Medusenform begrenzten Keimbahn zu sprechen. Der sukzessive, auf immer mehr I-Zellen übergreifende Potenzverlust, wie ihn die Deutung 1 annimmt, entspricht allerdings nicht ganz diesem Begriff in seiner bisherigen Bedeutung. 